\title{
Double-Diffusive of Natural Convection in an Inclined Porous Square Domain Generalized Model
}

\author{
Khaled Al-Farhany ${ }^{a, b *}$ and A. Turan ${ }^{b}$ \\ ${ }^{a}$ Department of mechanical engineering, University of Al-Qadisiyah, Al-Qadisiyah, Iraq. \\ ${ }^{b}$ School of Mechanical, Aerospace and Civil Engineering, the University of Manchester, P.O. Box 88, Manchester M60 1QD, UK
}

\section{ART ICLE INFO}

\section{Article history:}

Received 05 August 2019

Received in revised form 18 September 2019

Accepted 19 September 2019

\section{Keywords:}

Inclined cavity

Porous medium

Double diffusion

Natural convection

Generalized model

\begin{abstract}
A B S TR A C T
Numerical investigate of double-diffusive natural convection in an inclined porous square. Two opposing walls of the square cavity are adiabatic; while the other walls are, kept at constant concentrations and temperatures. The Darcy-Forchheimer-Brinkman model is used to solve the governing equations with the Boussinesq approximation. A code written in FORTRAN language developed to solve the governing equations in dimensionless forms using a finite volume approach with SIMPLER algorithm. The results presented in U-velocity and V-velocity, isotherms, iso-concentration, streamline, the average Nusselt number, and the average Sherwood number for different values of the dimensionless parameters. A wide range of these parameters have been used including; Darcy Number, modified Rayleigh number, Lewis number, buoyancy ratio, and inclination angle. The results show that for opposite buoyancy ratio $(\mathrm{N} \leqslant-1)$, the $\mathrm{Nu}$ decreases when the Le increases and the Sh increase when the Le increases. For an $(\mathrm{N}>0)$, the $\mathrm{Nu}$ increases when the Le increases until Le is equal to 1 and then it decreases, also Sh increases when the Le increases
\end{abstract}

\section{Introduction}

There are several important engineering applications for the convective heat and mass transfer on surfaces and rectangular porous cavities. Many studies presented in terms of uniform/non-uniform surface temperatures and/or heat flux Nield and Bejan [1].

A numerical and analytical investigation made by Vasseur et al. [2] on thin inclined porous layer to presented the effect of natural convective heat transfer by using a constant heat flux on two opposing walls. The buoyancy has driven the flow by using the Boussinesq approximation in a Darcy porous medium subjected to constant heat flux conditions. The successive over-relaxation method was used to solve the momentum equations and the energy equation was solved by using the alternating direction implicit method. The analytical solution was tested numerically in the range;
$20 \leq R \leq 50,0 \leq \phi \leq \pi$ and $2 \leq A \leq 10$. Later, the Vasseur et al.'s works have been extended by Sen et al. [3] to cover the flows which presented the unicellular convective motions only. Sen et al. studied a multiplicity of solutions for inclined porous enclosures. Their results presented in terms of small inclination angles and small range of Rayleigh number . Moya et al. [4], Caltagirone and Bories [5], and Báez and Nicolás [6] also studied the same cases. An experimental study of natural convective heat transfer in inclined porous layer by Inaba et al. [7]. In their studies, a tall rectangular cavity has been used. Two opposing walls were kept at different constant temperatures while the other walls were insulated. The experimental work covered a wide range of modified Prandtl number $3.1 \leq \operatorname{Pr} * \leq 499$, the modified Rayleigh number

* Corresponding author.

E-mail address: khaled.alfarhany@qu.edu.iq (Khaled AL-Farhany) 


\begin{tabular}{|llll|}
\hline \multicolumn{2}{l}{ Nomenclature } & & \\
$C$ & non-dimensional concentration, & $\mathrm{P}$ & non-dimensional pressure \\
$c p$ & specific heat at constant pressure & $\mathrm{Pr}$ & Prandtl number \\
$\mathrm{Da}$ & Darcy number, & $\mathrm{Ra}$ & modified Rayleigh number \\
$g$ & gravitational acceleration & $\mathrm{Sh}$ & average Sherwood numbers \\
$K$ & permeability of the porous medium & $\mathrm{T}$ & non-dimensional temperature \\
$k$ & thermal conductivity & Greek symbols \\
$L$ & length of the cavity & $\alpha$ & effective thermal diffusivity \\
$\mathrm{Le}$ & Lewis number & $\varepsilon$ & porosity \\
$N$ & buoyancy ratio & $\sigma$ & ratio of specific heats \\
$N u$ & average Nusselt number & $\phi$ & inclination angle \\
& & & \\
\hline
\end{tabular}

$34 * 10^{4}<R a^{*}<3.8 * 10^{4}$, the geometrical aspect ratio $5 \leq H / W \leq 32.7$, inclination angles $0 \leq \theta \leq 180$ and $0.074 \leq d / W \leq 1.0$. Four correlation equations for the natural heat transfer have been presented. Their results showed that; the maximum

Nusselt number occurs when the angle of inclination equal to $0^{\circ}$, for high Rayleigh numbers. Furthermore, the maximum Nusselt number occurs also, at low Rayleigh numbers when the angle of inclination equal to $60^{\circ}$. Another numerical study made by Hsiao [8] to presented the effects of the variable porosity on the natural heat transfer in an inclined porous enclosure. An important study of double-diffusive natural heat and mass transfer in an inclined porous enclosure, with the presence of temperaturedifference dependent heat generation made by Chamkha and Al-Mudhaf [9]. The results were presented for $(\mathrm{A}=2, \mathrm{Pr}=7.6$, and $\mathrm{Le}=10)$. In general, the results showed that; the Nusselt and Sherwood numbers decreases when the angle of inclination increases. However, there was an exception at a critical angle where the Nusselt and Sherwood numbers achieved the maximum magnitude. Wang et al. [10] studied the effect of time-periodic boundary conditions on inclined porous enclosure by using DarcyBrinkman model. Later, three-dimensional unsteady state natural convective heat transfer in an inclined porous cavity with time oscillating boundary conditions investigated numerically by Wang et al. [11]. The Darcy-Forchheimer-Brinkman model has been used in there study. Their results showed that, for a small inclination angle $\left(0^{\circ} \leq \alpha \leq 75^{\circ}\right)$, the natural convection inside the cavity almost stable and could being twodimensional. On the other hand, for large inclination angle $\left(75^{\circ} \leq \alpha \leq 90^{\circ}\right)$, the flow patterns inside are much more complicated and three-dimensional multiple roll-cells with different intercrossing angles are established. Many studies presented in Nield and Bejan [1], Ingham and Pop [12, 13], and Mojtabi and Charrier-Mojtabi [14] books in terms of double diffusion heat and mass transfer in saturated porous medium. Nithiarasu et al. [15, 16] studied numerically the double-diffusion natural convection by using NonDarcian models for opposing equal Buoyancy forces due to concentration and temperature. Many cases have been studied in terms of unsteady flow [17-21]. Also there are many studies of double diffusive natural convective in rectangular porous enclosure using Darcy model, effect of magnetic field, and source and sink heat for example [22]. Kefayati [23], [24] studied numerically the double diffusive natural convection flow in an inclined porous cavity for non-Newtonian power-law fluids flow. The Finite Difference Lattice Boltzmann Method has been used in this simulation. It was obtained that the Soret and Dufour parameters effect on the heat and mass and the entropy generation transfer considerably. Zhuang [25] studied numerically the double diffusive natural convective heat and mass transfer of power-law fluids in a cubic porous cavity with chemical reaction under the local thermal non-equilibrium. The generalized non-Darcy model has been used and the chemical reaction submitted to horizontal concentration and temperature gradients using the compact high order finite volume method.
To the authors' knowledge, double-diffusive natural convective heat and mass transfer in an inclined square porous medium, using the generalized model, has not been studied deeply. In this paper, the steady DoubleDiffusive Natural Convection in an Inclined Porous Square Domain Generalized Model are investigated. Two opposing walls of the square cavity are adiabatic, while the other walls are at constant concentrations and temperatures. AL-FARHANY code (written in FORTRAN language) have been used to solve the governing equations in dimensionless forms using finite volume approach with SIMPLER algorithm. The results are presented in term of Nusselt number and Sherwood number profiles as well as streamlines, isotherms and iso-concentration.

\section{Mathematical Modelling}

The geometrical model of the two-dimensional inclined square cavity filled with a homogeneous porous medium has been presented in Fig. 1. The walls assumed to be impervious. Two opposing walls of the square enclosure are adiabatic; while the other walls are, have constant concentrations and temperatures. The medium is assumed to be incompressible, isotropic, and homogeneous.

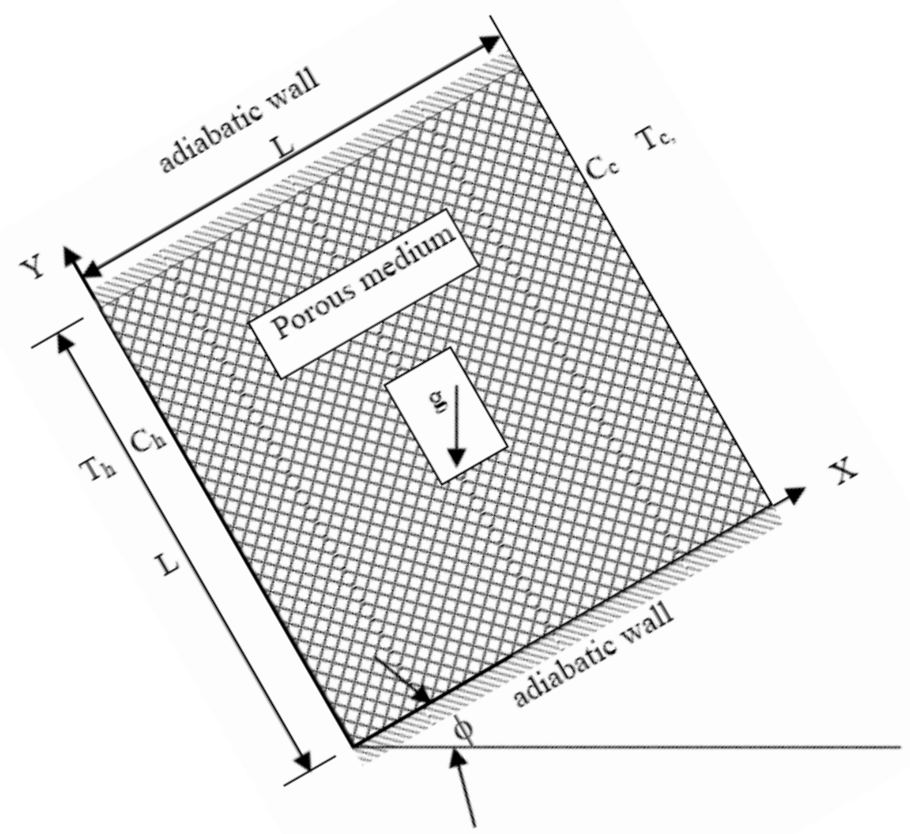

Figure 1. Scheme of the model

The governing equations in terms of non-dimensional form are: [1]:

$$
\rho=\rho\left[1-\beta_{T}\left(T-T_{o}\right)-\left[1-\beta_{c}\left(C-C_{o}\right)\right]\right]
$$


Dimensionless continuity equation:

$$
\frac{\partial U}{\partial X}+\frac{\partial V}{\partial Y}=0
$$

Dimensionless momentum equation in $\mathrm{X}$-axis:

$$
\begin{aligned}
& \frac{1}{\varepsilon^{2}} U \cdot \frac{\partial U}{\partial X}+\frac{1}{\varepsilon^{2}} V \cdot \frac{\partial U}{\partial Y}= \\
& -\frac{\partial P}{\partial X}-\frac{P r}{D a} \cdot U-\frac{1.75}{\sqrt{150}} \cdot \frac{\sqrt{U^{2}+V^{2}}}{\sqrt{D a}} \cdot \frac{U}{\varepsilon^{3 / 2}}+\frac{P r}{\varepsilon}\left(\frac{\partial^{2} U}{\partial X^{2}}+\frac{\partial^{2} U}{\partial Y^{2}}\right)
\end{aligned}
$$$$
+R a \cdot \operatorname{Pr} \cdot T \cdot \sin (\phi)+N \cdot R a \cdot \operatorname{Pr} \cdot C \cdot \sin (\phi)
$$

Dimensionless momentum equation in $\mathrm{Y}$-axis:

$$
\begin{aligned}
& \frac{1}{\varepsilon^{2}} U \cdot \frac{\partial V}{\partial X}+\frac{1}{\varepsilon^{2}} V \cdot \frac{\partial V}{\partial Y}= \\
& -\frac{\partial P}{\partial Y}-\frac{P r}{D a} \cdot V-\frac{1.75}{\sqrt{150}} \cdot \frac{\sqrt{U^{2}+V^{2}}}{\sqrt{D a}} \cdot \frac{V}{\varepsilon^{3 / 2}}+\frac{\operatorname{Pr}}{\varepsilon}\left(\frac{\partial^{2} V}{\partial X^{2}}+\frac{\partial^{2} V}{\partial Y^{2}}\right)
\end{aligned}
$$$$
+R a \cdot \operatorname{Pr} \cdot T \cdot \cos (\phi)+N \cdot R a \cdot \operatorname{Pr} \cdot C \cdot \cos (\phi)
$$

Dimensionless energy equation:

$$
U \cdot \frac{\partial T}{\partial X}+V \cdot \frac{\partial T}{\partial Y}=\frac{\partial^{2} T}{\partial X^{2}}+\frac{\partial^{2} T}{\partial Y^{2}}
$$

Dimensionless species equation:

$$
U \cdot \frac{\partial C}{\partial X}+V \cdot \frac{\partial C}{\partial Y}=\frac{\partial^{2} C}{L e^{*} \partial X^{2}}+\frac{\partial^{2} C}{L e^{*} \partial Y^{2}}
$$

The average Nusselt number ( $N \boldsymbol{u}$ ) and Sherwood number $(S h)$ at the walls can be calculated as:

$$
\begin{aligned}
& \left.N u=\int_{0}^{1}-\frac{\partial T}{\partial X}\right) \cdot \partial Y \\
& \left.S h=\int_{0}^{1}-\frac{\partial C}{\partial X}\right) \cdot \partial Y
\end{aligned}
$$

The Non-dimensional parameters are:

$$
\begin{aligned}
& \mathrm{Y}= \frac{\mathrm{y}}{\mathrm{L}}, \mathrm{X}=\frac{\mathrm{x}}{\mathrm{L}}, \quad U=\frac{u L}{\alpha}=-\frac{\partial \psi}{\partial Y}, V=\frac{v L}{\alpha}=\frac{\partial \psi}{\partial X}, P r=\frac{v}{\alpha}, \\
& P= \frac{p L^{2}}{\rho \alpha^{2}} \mathrm{Ra}=\frac{g \beta_{T} \Delta T L^{3}}{v \alpha}, L e=\frac{\alpha}{D_{c}}, N=\frac{\beta_{c} \Delta C}{\beta_{T} \Delta T} \\
& k_{e f f}=\varepsilon k_{f}+(1-\varepsilon) k_{s}, \quad D a=\frac{K}{L^{2}}, \alpha=\frac{k_{e f f}}{\left(\rho \cdot c_{p}\right)_{f}}, \\
& T=\frac{\bar{T}-\bar{T}_{c}}{\overline{T_{h}}-\bar{T}_{c}}, C=\frac{\bar{C}-\bar{C}_{c}}{\bar{C}_{h}-\bar{C}_{c}}, \\
& \sigma=\frac{\varepsilon\left(\rho \cdot c_{p}\right)_{f}+(1-\varepsilon)\left(\rho \cdot c_{p}\right)_{s}}{\left(\rho \cdot c_{p}\right)_{f}}
\end{aligned}
$$

The Non-dimensional initial boundary conditions are:

$$
\begin{aligned}
& \text { at } X=0, \text { and any } Y ; \quad U=V=0 ; \quad C_{h}=T_{h}=1 \\
& \text { at } X=1, \text { and any } Y ; \quad U=V=0 ; \quad C_{c}=T_{c}=0
\end{aligned}
$$

at $Y=0,1$, and $X ; U=V=0 ; \frac{\partial T}{\partial Y}=\frac{\partial C}{\partial Y}=0$

\section{Solution Procedure}

In this study, the non-dimensional governing equations solved by using the finite volume methods. The SIMPLER algorithm has been chosen for the pressure velocity coupling. A second order central discretization scheme is used for the energy, species and momentum equations. The mesh independent approach has been used to choose the optimum grid size, which was 120X120. Al-FARHANY code has been used in this study and it was validated using previous studies (Al-Farhany and Turan [26-28]).

\section{Results}

The results presented in terms of streamlines, isotherms, and isoconcentration, U-velocity, V-velocity, as well as the average Sherwood number and Nusselt number profiles. The Prandtl number is taken as unity. Fig. 2 shows the stream functions, isotherms and iso-concentration lines for $D a=10^{-6}, R a^{*}=100$, and $L e=10.0$ where $N=-1.0$, with different inclination angles $(\phi)$. Where there is no inclination angle $\left(\phi=0^{\circ}\right)$, the secondary circulations appear and the counter clockwise flow is homogenised and symmetric on the main diagonal of the porous cavity. This happens because the buoyancy ratio, which is a ratio of fluid density contributions by the concentration to the temperature variations, is equal to -1 . The maximum positive stream functions are about 1.20 and the maximum negative stream functions were about -0.7. It is observed that the variations of isotherms near the insulated walls are higher than in the middle of the cavity. For the left half of the cavity $(0<X<0.5)$, and near the left bottom corner, it is observed that the isotherms are increased in the isotherms up to that are near the middle of the cavity height and then decreased until the left top corner. This behaviour occurred as a result of the fluid rising due to the influence of the buoyancy force and the effect of the secondary circulations. It is clear that the maximum isotherms are not in the middle of the cavity length but they are almost near places where there is no movement in the fluid flow (when the stream functions are equal to zero). The same behaviour could be seen in the left half of the cavity but in the opposite direction. Also, for the iso-concentration, it could be seen that the isoconcentration field is more sensitive than the isotherms and that occurred due to the considered Lewis number ( $L e=10)$. The maximum (minimum) iso-concentration appeared near the left (right) wall and around the maximum positive stream functions and most of the iso-concentration variations were near the maximum negative stream functions which are around the main diagonal of the cavity. By increasing the angle of inclination $\left(\phi>0^{\circ}\right)$, it is observed that the positive vortex strength increased when $(\phi)$ is increased until $\phi=60^{\circ}$ and after that the positive vortex strength decreased with increasing of $(\phi)$. Also, the left positive secondary circulations decreased until they disappeared. For $\phi=45^{\circ}$, the maximum positive stream functions and the maximum negative stream functions were about 3.85 and -0.29 respectively. Furthermore, the negative vortex strength is decreased when $\phi$ is increased to around $45^{\circ}$ and then it increased with the increasing of $(\phi)$. As noted above, the maximum iso-concentration variations are near the maximum negative stream functions and the iso- 
concentration lines are almost parallel close to the left high concentration wall and then increased in the iso-concentration fields near the insulated top wall.

This behaviour is clearly observed with an increase of the angle of inclination for both isotherm and iso-concentrations' fields. For cases of $\phi=90^{\circ}$, different behaviours appeared. It is observed that no vortex appeared and that happened because of low flow intensity. The heat was transfer through the porous cavity by conduction instead of natural convection.

For a low Lewis number, Fig. 3 shows the stream functions, isotherms and iso-concentration lines for $D a=10^{-6}, R a^{*}=100, \operatorname{Pr}=1$, and $L e=0.1$ with different inclination angles $(\phi)$ where $N=-1.0$. For a low Lewis number, the flow has almost been driven due to mass transfer rather than heat transfer. When the buoyancy ratio is equal to -1 , Fig. 3 shows that no significant heat and mass transfer appears for a low inclination angle especially when the inclination angle is equal to zero $\left(\phi=0^{\circ}\right)$. From the stream function lines, it is clear that the vortex strength of the fluid is very small. Furthermore, the concentration boundary layer is nearly equal to the isotherms' boundary layer and it clearly can be seen that the isotherm and iso-concentration lines are parallel and both the Nusselt and Sherwood numbers are equal to 1. For $\phi>0^{\circ}$, it can be observed that the positive vortex strength increases when the angle of the inclination is increased until $\phi=60^{\circ}$ and subsequently the positive vortex strength decreases when the angle of the inclination is increased. Also, the negative secondary circulations decrease until they disappear. For $\phi=45^{\circ}$, the maximum positive stream functions and the maximum negative stream functions are about 2.0 and -0.04 respectively. The maximum isotherms' boundary layer can be seen at $\phi=60^{\circ}$; therefore the Nusselt number is also at its maximum at $\phi=60^{\circ}$, as shown in Table 1. For the cases of heated and concentrated from below $\left(\phi=90^{\circ}\right)$, a multiplicity of solutions happened (monocellular and bicellular flows), most of these solution results are in bicellular flow as shown in the Fig. 3. For Le=0.1, the bicellular flow solution was chosen for all the cases of $\phi=90^{\circ}$. In the case of the isotherms, it is observed that the isotherms' boundary layers near the insulated walls are thinner than the isotherms' boundary layers near the middle of the bottom wall. Because of this, the fluid flows in the porous cavity rise up from the middle of the hot bottom wall towards the cold top wall. As a result of the effects of the thick cold boundary layer near the top wall, the vertical fluid flow velocities decreased until they are at a minimum near the cold wall, subsequently, the fluid flow was driven in two different horizontal directions to achieve the bicellular flows. For a high buoyancy ratio, Fig. 4 shown the stream functions, iso-concentration and isotherms for $D a=10^{-6}, R a^{*}=100, P r=1$, and $L e=0.1$ with different inclination angles ( $\phi$ ) where $N=+5.0$. A high positive buoyancy ratio means that the fluid rises up due to the influence of the temperature and mass variations which is more than the effect of the temperature variations in the buoyancy force. The results clearly show that when the angle of the inclination increases, the vortex strength of the fluid increases until $\phi=45^{\circ}$ that subsequently the vortex strength decreases when the $(\phi)$ increases. It can also be observed that there is only a main clockwise flow (no secondary flow) at a maximum positive stream function of about 34.65 at $\phi=45^{\circ}$. Moreover, the isotherms' boundary layer is too thin near the hot wall and it increases near the left upper corner. Also, it is very clear that the isotherms are more sensitive than the iso-concentrations and this happens due to the considered Lewis number $(L e=0.1)$. In the case of a low Lewis number the effect of the inclination angles are not significant in either the isotherm or the iso-concentrations' field. As mentioned previously, when $\phi=90^{\circ}$ bicellular flows occur. Also, it is observed that the isotherms' boundary layers near the insulated walls are thicker than the isotherms' boundary layers near the middle of the bottom wall. This is because the fluid flows in the porous cavity rise up near the insulated walls from the hot bottom wall towards the cold top wall. As a result of the effects of the thick cold boundary layer near the top wall, the vertical fluid flow velocities decrease until they are at a minimum near the cold wall; on the other hand, the horizontal velocity increases and then decreases to achieve minimum velocity near the middle of the cavity in order to achieve the bicellular flows. Fig. 5 shows the vertical/horizontal velocity variations on the middle of the vertical/horizontal planes of the cavity at $R a^{*}=500, D a=10^{-6}, N=2.0, L e=0.1$, and at different inclination angles. The $\mathrm{U}$ and $\mathrm{V}$ velocities increasing when the $(\phi)$ increasing until $\phi=60^{\circ}$ and then it decreases when $(\phi)$ increases. For the opposed flow, Fig. 6 presented the variation of the $\mathrm{U}$ and $\mathrm{V}$ velocity at the mid of the horizontal/vertical planes of the enclosure at $\mathrm{Ra}^{*}=500, \mathrm{Da}=10^{-6}, \mathrm{~N}=-5.0$, $\mathrm{Le}=1$ and different inclination angles. The $\mathrm{U}$-velocity profiles show that for $\phi=0^{\circ}$, the U-velocity profile is very sharp and it smoothly decreases when $(\phi)$ increases. In the case of the V-velocity profile, the V-velocity decreases when the angles of inclination increase. Also, it can be clearly observed that most of the high negative V-velocities are near the hot wall, the high positive $\mathrm{V}$-velocities are near the cold wall (counter clockwise flow) and the minimum $\mathrm{V}$-velocities are in the middle of the porous cavity.

The effect of the Rayleigh number on the average of Nussselt and Sherwood numbers is presented in Figs 7 (a) and 8 (b) respectively for $D a=10^{-4}$ and $\phi=30^{\circ}$ with a different Lewis and buoyancy ratio. These graphs show that, when the $\mathrm{Ra}$ increases when $\mathrm{Nu}$ increases. Additionally, for a positive buoyancy ratio, the $\mathrm{Nu}$ increases with increasing of Lewis number until $L e=1$ and then its decreases. Moreover, it decreases with increasing of Lewis number for a negative buoyancy ratio as shown in Table 1. At a constant $\mathrm{Ra}$, the reduction of the Lewis number naturally implies a relatively high mass diffusivity value. When the Le is decreased, the thickness of the thermal boundary layers near the hot and cold walls becomes thinner than the concentration boundary layers. Therefore, when the Lewis number decreases, the heat transfer increases and the mass transfer is decreased. Table 1 presents the effect of inclination angles on the Sherwood and Nusselt numbers for $R a^{*}=10^{-2}, D a=10^{-6}$, at different $L e$ and $N$. It shows that for a positive buoyancy ratio, the maximum average of Sh and $\mathrm{Nu}$ occur when the inclination angle is between $30^{\circ}$ and $45^{\circ}$. This means both of them increases when the $(\phi)$ increases until around $\phi=45^{\circ}$, then they decreasing with increasing of $(\phi)$. For the opposing flow (negative buoyancy ratio), both of the $N u$ and $S h$ decrease when $(\phi)$ decrease. 

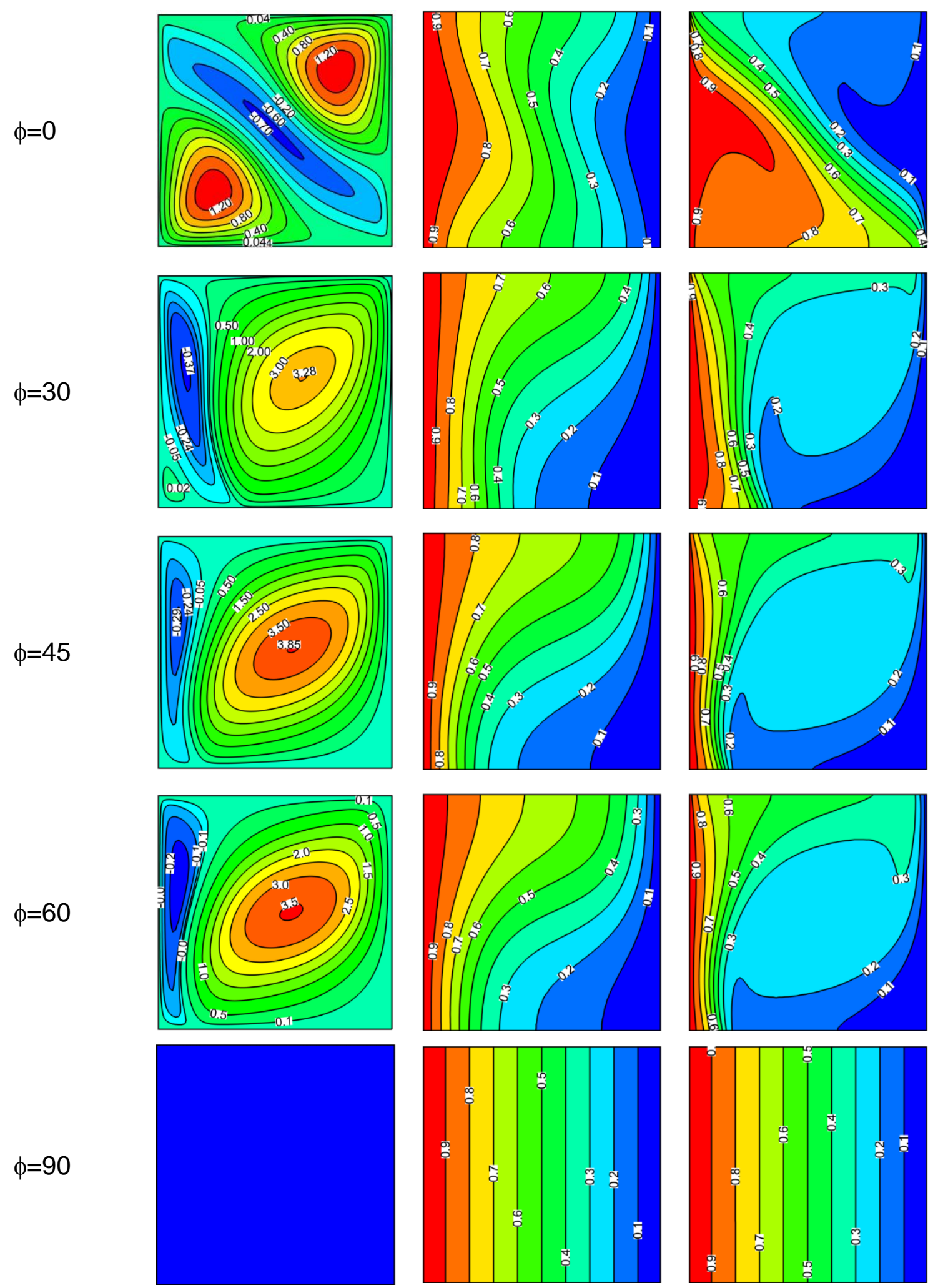

Figure 2. Stream functions (left), isotherms (middle) and iso-concentration (right) lines for $\operatorname{Da}=10^{-6}, \operatorname{Ra}^{*}=100, \operatorname{Pr}=1.0, \varepsilon=0.36, \mathrm{Le}=10.0$ and $\mathrm{N}=\mathbf{- 1 . 0}$ with different inclination angles. 

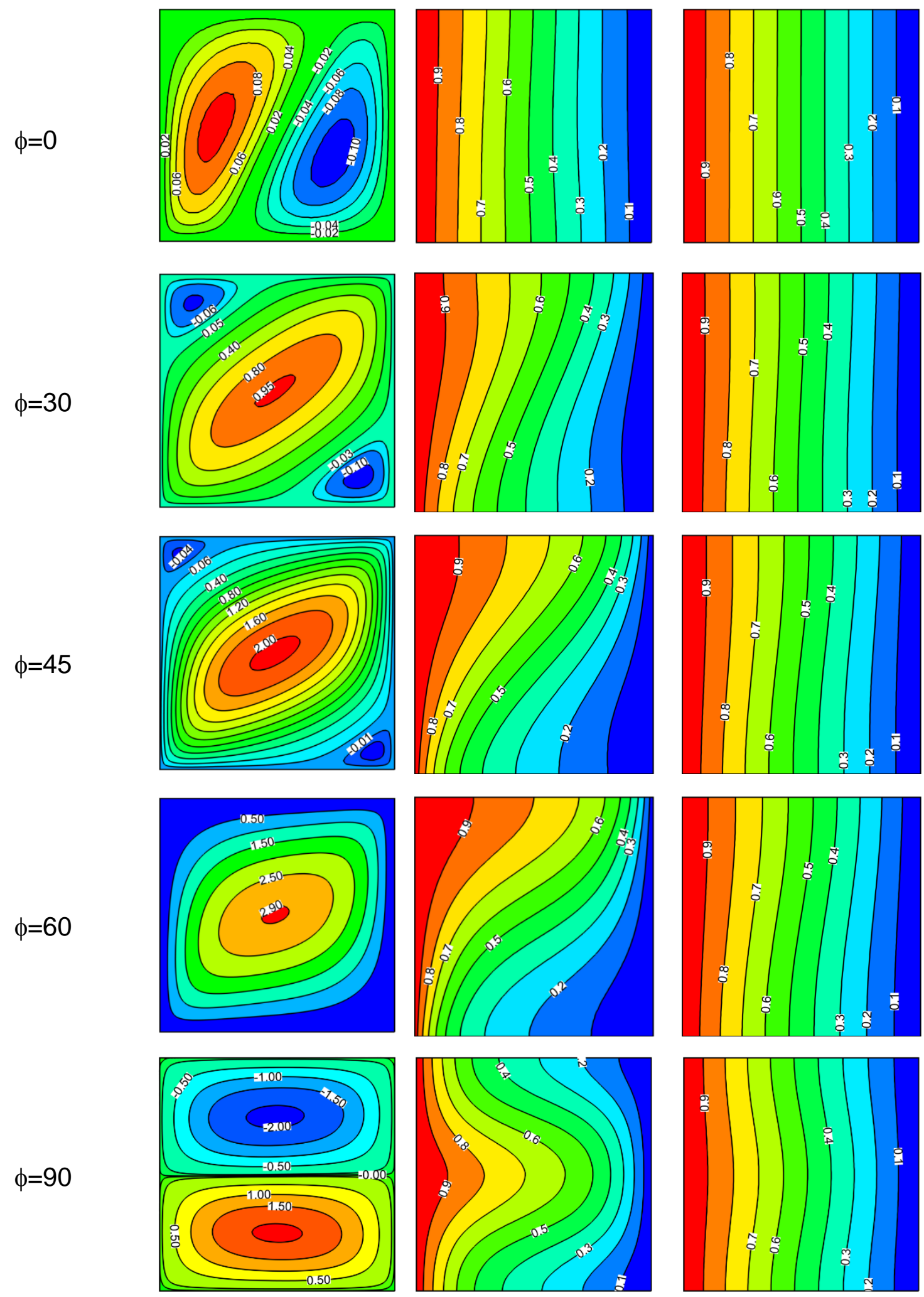

Figure 3. Stream functions (left), isotherms (middle) and iso-concentration (right) lines for $\operatorname{Da}=10^{-6}, \operatorname{Ra}{ }^{*}=100, \operatorname{Pr}=1.0, \varepsilon=0.36, \mathrm{Le}=0.1$ and $\mathbf{N}=-\mathbf{1 . 0}$ with different inclination angles. 

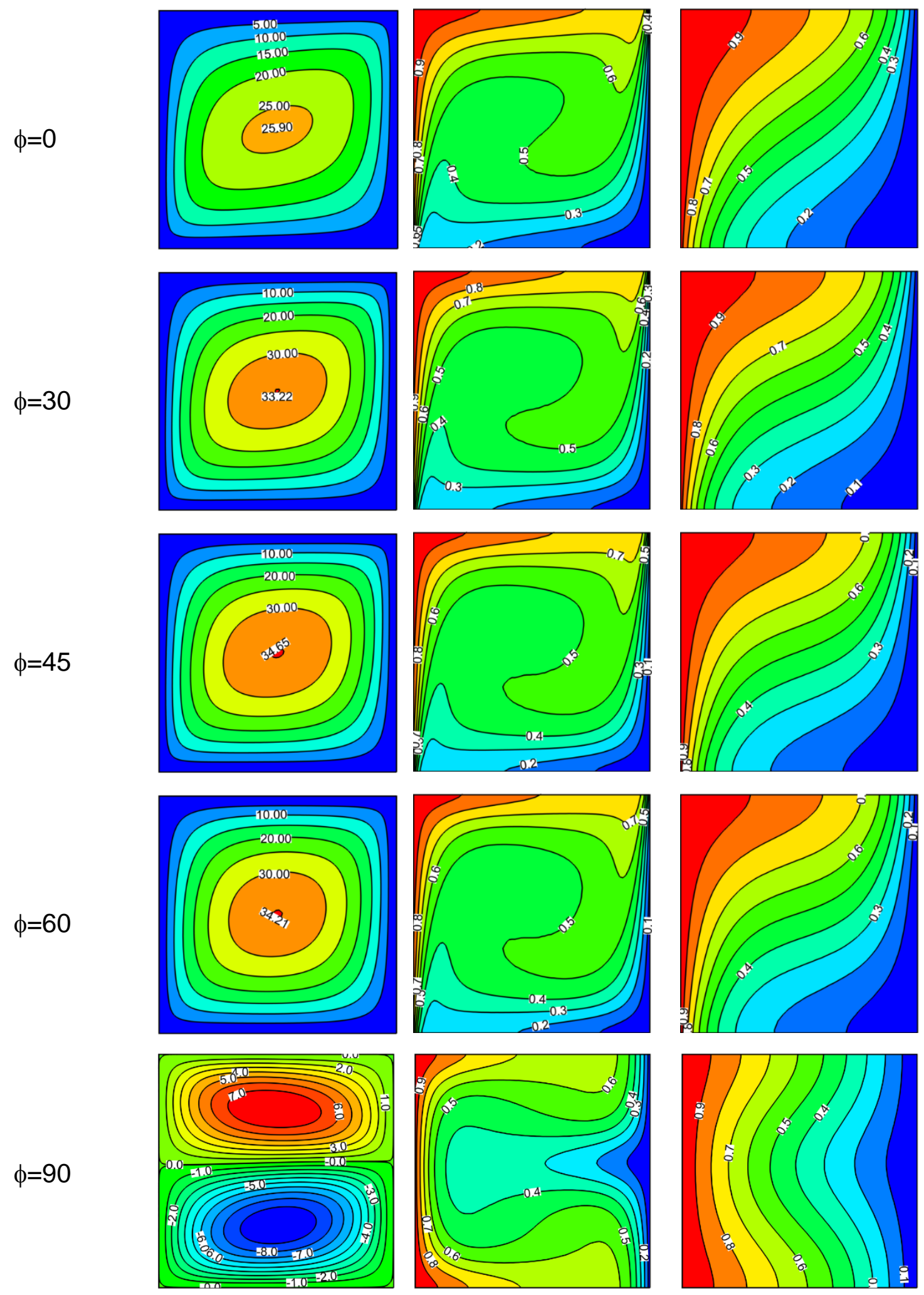

Figure 4. Stream functions (left), isotherms (middle) and iso-concentration (right) lines for $D a=10^{-6}, \operatorname{Ra}^{*}=100, \operatorname{Pr}=1.0, \varepsilon=0.36, \mathrm{Le}=0.1$ and $\mathrm{N}=\mathbf{5 . 0}$ with different inclination angles. 

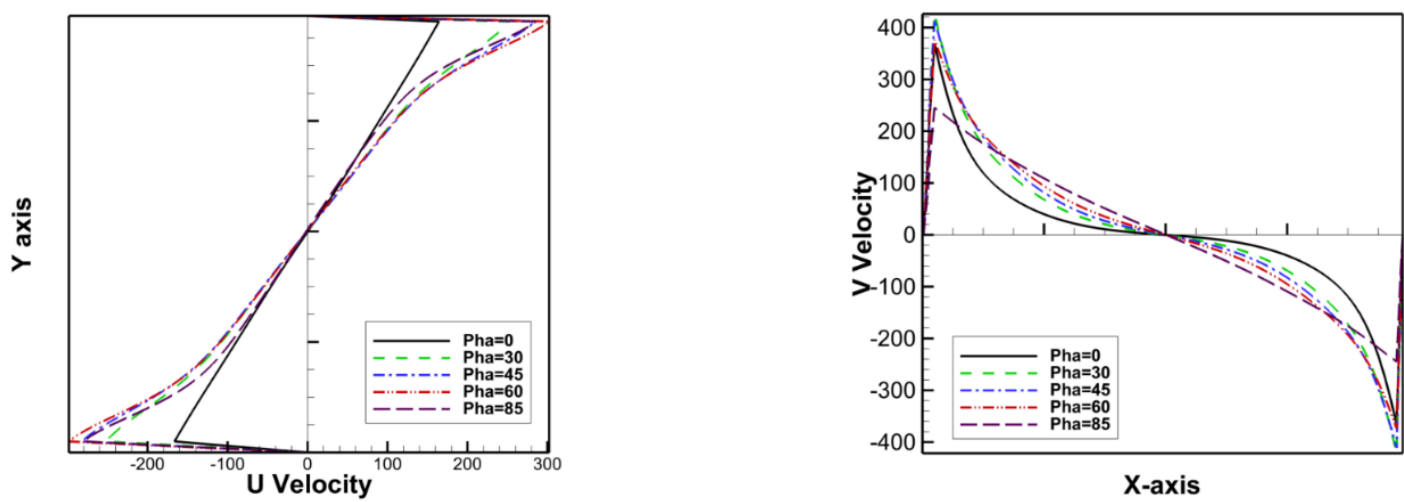

Figure $5 \mathrm{U}$-velocity at $\mathrm{X}=0.5$ and $\mathrm{V}$-velocity at $\mathrm{Y}=0.5$ for $\mathrm{Da}=10^{-6}$, $\mathrm{Ra}^{*}=500, \mathrm{Le}=\mathbf{0 . 1}, \mathrm{N}=\mathbf{2 . 0}$ for different inclination angles.
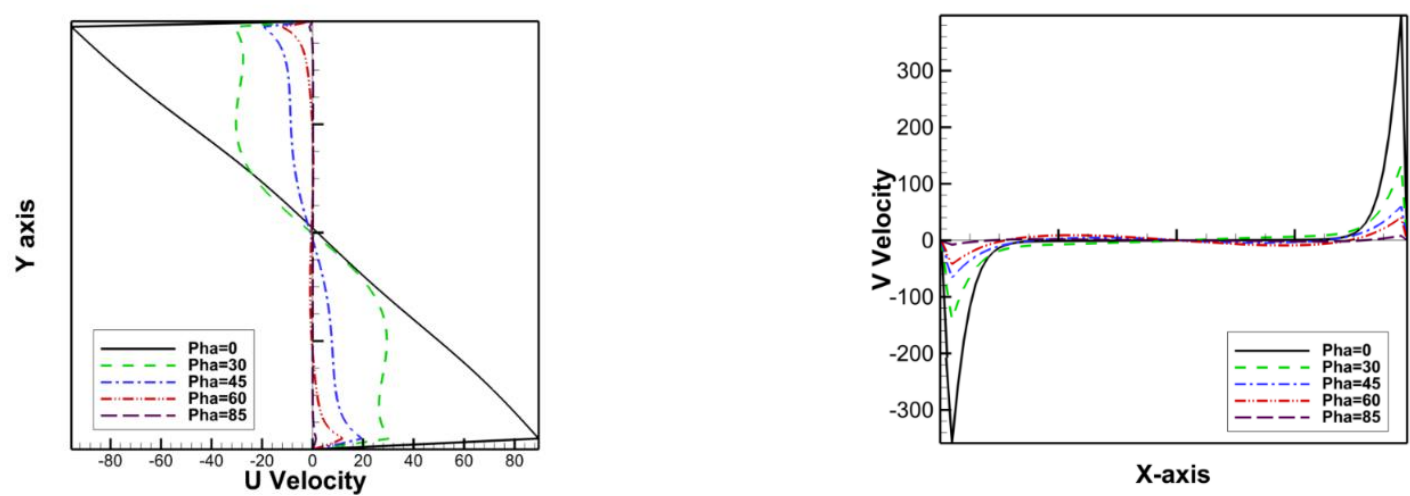

Figure $6 \mathrm{U}$-velocity at $\mathrm{X}=0.5$ and $\mathrm{V}$-velocity at $\mathrm{Y}=0.5$ for $\mathrm{Da}=10^{-6}, \mathrm{Ra} *=500, \mathrm{Le}=1.0, \mathrm{~N}=\mathbf{- 5 . 0}$ for different inclination angles.

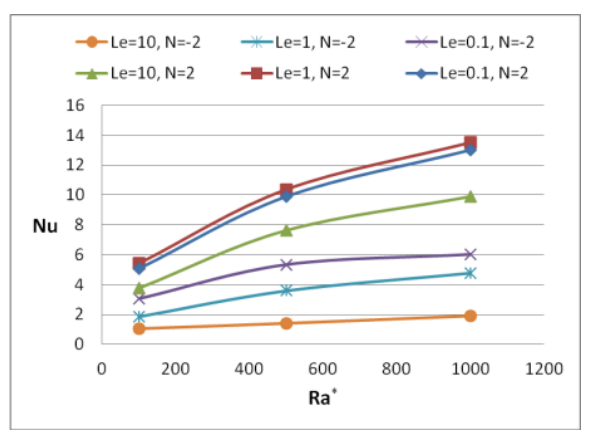

a

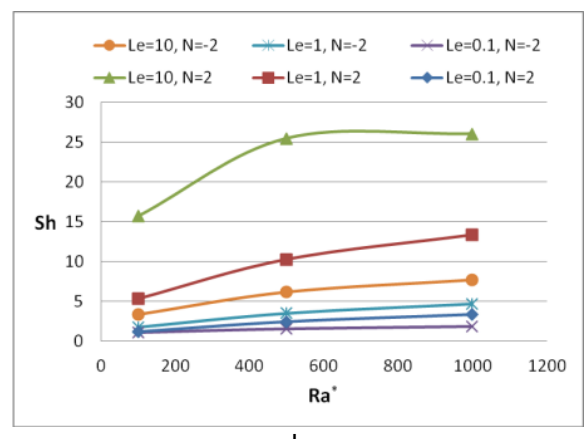

b

Figure 7 Variation of (a) average Nusselt numbers (Nu) and (b) average Sherwood (Sh) numbers with $\mathrm{Ra}^{*}$ for $\mathrm{Da}=10^{-4}, \phi=30^{\circ}$ with different Le and $N$. 
Table 1 Average Nusselt and Sherwood numbers at $\mathrm{Da}=10^{-6}, \mathrm{Ra}^{*}=10^{2}$

\begin{tabular}{|c|c|c|c|c|c|c|c|c|c|c|c|}
\hline \multirow{2}{*}{ Le } & \multirow{2}{*}{$N$} & \multicolumn{2}{|l|}{$\phi=0$} & \multicolumn{2}{|l|}{$\phi=30$} & \multicolumn{2}{|l|}{$\phi=45$} & \multicolumn{2}{|l|}{$\phi=60$} & \multicolumn{2}{|l|}{$\phi=85$} \\
\hline & & $\mathrm{Nu}$ & Sh & $\mathrm{Nu}$ & Sh & $\mathrm{Nu}$ & Sh & $\mathrm{Nu}$ & Sh & $\mathrm{Nu}$ & $\mathrm{Sh}$ \\
\hline & 5 & 9.47 & 1.98 & 10.15 & 2.31 & 9.88 & 2.33 & 9.15 & 2.25 & 6.65 & 1.80 \\
\hline & 2 & 6.46 & 1.34 & 6.80 & 1.46 & 6.54 & 1.45 & 6.00 & 1.38 & 4.17 & 1.16 \\
\hline \multirow[t]{5}{*}{0.1} & -1 & 1.00 & 1.00 & 1.12 & 1.00 & 1.54 & 1.01 & 1.97 & 1.01 & 2.33 & 1.017 \\
\hline & -2 & 3.60 & 1.17 & 3.40 & 1.13 & 3.21 & 1.10 & 2.95 & 1.07 & 2.27 & 1.018 \\
\hline & -5 & 7.61 & 1.84 & 6.13 & 1.47 & 5.26 & 1.30 & 4.33 & 1.16 & 2.18 & 1.02 \\
\hline & 5 & 9.12 & 9.12 & 10.66 & 10.66 & 10.47 & 10.46 & 9.66 & 9.65 & 7.93 & 7.93 \\
\hline & 2 & 6.30 & 6.29 & 7.48 & 7.47 & 7.36 & 7.36 & 6.85 & 6.86 & 5.02 & 5.02 \\
\hline \multirow[t]{5}{*}{1} & -1 & 1.00 & 1.00 & 1.00 & 1.00 & 1.00 & 1.00 & 1.00 & 1.00 & 1.00 & 1.00 \\
\hline & -2 & 3.11 & 3.08 & 2.01 & 1.98 & 1.54 & 1.52 & 1.24 & 1.21 & 1.02 & 1.01 \\
\hline & -5 & 7.43 & 7.42 & 4.06 & 4.05 & 2.34 & 2.33 & 1.44 & 1.44 & 1.02 & 1.01 \\
\hline & 5 & 3.90 & 19.60 & 5.50 & 23.22 & 5.88 & 22.86 & 5.84 & 20.8 & 5.84 & 20.8 \\
\hline & 2 & 3.58 & 17.87 & 4.86 & 19.49 & 5.03 & 19.28 & 4.91 & 18.47 & 3.35 & 17.02 \\
\hline \multirow[t]{3}{*}{10} & -1 & 1.17 & 4.29 & 1.52 & 3.04 & 1.79 & 3.70 & 1.65 & 3.42 & 1.00 & 1.00 \\
\hline & -2 & 1.47 & 10.10 & 1.07 & 3.94 & 1.01 & 1.77 & 1.00 & 1.20 & 1.00 & 1.0 \\
\hline & -5 & 2.56 & 16.14 & 1.31 & 6.74 & 1.07 & 2.89 & 1.01 & 1.52 & 1.00 & 1.00 \\
\hline
\end{tabular}

\section{Conclusions}

In this study, a generalized model used in a numerical investigation on double-diffusive natural convection in an inclined square cavity filled with porous medium. Two opposing walls of the square cavity are adiabatic, while the other walls are at constant concentrations and temperatures. ALFARHANY code has been used to solve the governing equations in dimensionless forms using finite volume approach with SIMPLER algorithm. The results are presented in terms of $\mathrm{Sh}$ and $\mathrm{Nu}$ profiles, and also for the streamline, iso-concentration, and isothermal. The predictions included different values in the dimensionless parameters, specifically, cavity inclination angle $\left(0^{\circ} \leq \phi \leq 90^{\circ}\right)$, buoyancy ratio $(-5 \leq N \leq 5)$, Lewis number $(0.1 \leq L e \leq 10)$, Darcy number $\left(10^{-6} \leq D a \leq 10^{-2}\right)$ and Rayleigh number $\left(100 \leq R a^{*} \leq 1000\right)$, while the Prandtl number is taken as $\operatorname{Pr}=1.0$. The results show that, when opposite buoyancy forces are considered $(N \leq$ 1 ), the convection in the porous cavity is always a multiplicity of steady solutions with flow fields of two, three and four flow cells. Also, it can be observed that the positive vortex strength increases when the angle of the inclination is increased until $\phi=45^{\circ}$ and subsequently the positive vortex strength decreases when the angle of the inclination is increased. Also, the same behaviour can be seen for the positive buoyancy forces. Furthermore, the negative vortex strength decreases when the angle of the inclination is increased. For cases of $\phi=90^{\circ}$, it can be observed that no vortex appears and this occurs because of the low flow intensity. On the other hand, when a positive buoyancy ratio is considered the convection in the porous cavity is always in a single cell and it occurs in a clockwise flow. Moreover, at $\phi=90^{\circ}$ the bicellular flows occur.

In general, the results show that for a positive buoyancy ratio $(N>0)$, the $\mathrm{Nu}$ and Sh increase when the angle of inclination increases until around $\phi=45^{\circ}$, and then they decrease when $\phi$ is increased. For an opposing flow (negative buoyancy ratio), both the $\mathrm{Nu}$ and $\mathrm{Sh}$ decrease when the angle of inclination is decreased. Also, the average Sherwood and Nusselt numbers increase when the Rayleigh number increases, while they decrease when the Darcy number increases. For an $(N>0)$, the Nu increases when the Le increases until Le is equal to 1 and then the $N u$ decreases when the $L e$ increases. On the other hand, the $N u$ decreases when the $L e$ increases for a negative buoyancy ratio. Moreover, the Sherwood number increases when the Lewis number increases. It was also observed that the $\mathrm{Nu}$ and $\mathrm{Sh}$ numbers increase when the $|N|>-1$ increases.

\section{REFERENCES}

[1] Nield, D.A. and A. Bejan, Convection in Porous Media [electronic resource]. Third Edition. ed. 2006, New York, NY: Springer Science+Business Media, Inc. digital.

[2] Vasseur, P., M.G. Satish, and L. Robillard, Natural convection in a thin, inclined, porous layer exposed to a constant heat flux. International Journal of Heat and Mass Transfer, 1987. 30(3): p. 537-549.

[3] Sen, M., P. Vasseur, and L. Robillard, Multiple steady states for unicellular natural convection in an inclined porous layer. International Journal of Heat and Mass Transfer, 1987. 30(10): p. 2097-2113.

[4] Moya, S.L., E. Ramos, and M. Sen, Numerical study of natural convection in a tilted rectangular porous material. International Journal of Heat and Mass Transfer, 1987. 30(4): p. 741-756.

[5] Caltagirone, J.P. and S. Bories, Solutions and stability criteria of natural convective flow in an inclined porous layer. Journal of Fluid Mechanics, 1985. 155: p. 267-287.

[6] Báez, E. and A. Nicolás, Natural convection fluid flow and heat transfer in porous media. Journal of Mechanics of Materials and Structures, 2007. 2(8): p. 1571-1584.

[7] Inaba, H., M. Sugawara, and J. Blumenberg, Natural convection heat transfer in an inclined porous layer. International Journal of Heat and Mass Transfer, 1988. 31(7): p. 1365-1374.

[8] Hsiao, S.W., Natural convection in an inclined porous cavity with variable porosity and thermal dispersion effects. International Journal of Numerical Methods for Heat and Fluid Flow, 1998. 8(1): p. 97-117.

[9] Chamkha, A.J. and A. Al-Mudhaf, Double-diffusive natural convection in inclined porous cavities with various aspect ratios and temperature-dependent heat source or sink. Heat and Mass Transfer/Waerme- und Stoffuebertragung, 2008. 44(6): p. 679-693.

[10] Wang, G., et al., Numerical study of natural convection heat transfer in an inclined porous cavity with time-periodic boundary conditions. Transport in Porous Media, 2008. 74(3): p. 293-309.

[11] Wang, Q.W., et al., Three-dimensional numerical study of natural convection in an inclined porous cavity with time sinusoidal oscillating boundary conditions. International Journal of Heat and Fluid Flow, 2010. 31(1): p. 70-82.

[12] Ingham, D.B. and I. Pop, Transport Phenomena in Porous Media I. 2000: UK: Pergamon/Elsevier Science, .

[13] Ingham, D.B. and I. Pop, Transport Phenomena in Porous Media 
III. 2005: UK: Pergamon/Elsevier Science, .

[14] Mojtabi, A. and M.C. Charrier-Mojtabi, Double-diffusive convection in porous media in Handbook of Porous Media, chapter 7. Marcel Dekker, New York,. 2005.

[15] Nithiarasu, P., T. Sundararajan, and K.N. Seetharamu, Doublediffusive natural convection in a fluid saturated porous cavity with a freely convecting wall. International Communications in Heat and Mass Transfer, 1997. 24(8): p. 1121-1130.

[16] Nithiarasu, P., K.N. Seetharamu, and T. Sundararajan, Non-Darcy double-diffusive natural convection in axisymmetric fluid saturated porous cavities. Heat and Mass Transfer/Waerme- und Stoffuebertragung, 1997. 32(6): p. 427-433.

[17] Nishimura, T., M. Wakamatsu, and A.M. Morega, Oscillatory double-diffusive convection in a rectangular enclosure with combined horizontal temperature and concentration gradients. International Journal of Heat and Mass Transfer, 1998. 41(11): p. 1601-1611.

[18] Weaver, J.A. and R. Viskanta, Natural convection in binary gases driven by combined horizontal thermal and vertical solutal gradients. Experimental Thermal and Fluid Science, 1992. 5(1): p. 57-68.

[19] Trevisan, O.V. and A. Bejan, Natural convection with combined heat and mass transfer buoyancy effects in a porous medium. International Journal of Heat and Mass Transfer, 1985. 28(8): p. 1597-1611.

[20] Goyeau, B., J.P. Songbe, and D. Gobin, Numerical study of doublediffusive natural convection in a porous cavity using the DarcyBrinkman formulation. International Journal of Heat and Mass Transfer, 1996. 39(7): p. 1363-1378.

[21] Nithiarasu, P., K.N. Seetharamu, and T. Sundararajan, Doublediffusive natural convection in an enclosure filled with fluidsaturated porous medium: A generalized non-Darcy approach. Numerical Heat Transfer; Part A: Applications, 1996. 30(4): p.
413-426.

[22] Kefayati, G., Simulation of double diffusive $M H D$ (magnetohydrodynamic) natural convection and entropy generation in an open cavity filled with power-law fluids in the presence of Soret and Dufour effects (part II: entropy generation). Energy, 2016. 107: p. 917-959.

[23] Kefayati, G.R., Simulation of double diffusive natural convection and entropy generation of power-law fluids in an inclined porous cavity with Soret and Dufour effects (Part I: Study of fluid flow, heat and mass transfer). International Journal of Heat and Mass Transfer, 2016. 94: p. 539-581.

[24] Kefayati, G.R., Simulation of double diffusive natural convection and entropy generation of power-law fluids in an inclined porous cavity with Soret and Dufour effects (Part II: Entropy generation). International Journal of Heat and Mass Transfer, 2016. 94: p. 582624.

[25] Zhuang, Y., H. Yu, and Q. Zhu, A thermal non-equilibrium model for 3D double diffusive convection of power-law fluids with chemical reaction in the porous medium. International Journal of Heat and Mass Transfer, 2017. 115: p. 670-694.

[26] Al-Farhany, K. and A. Turan, Numerical study of double diffusive natural convective heat and mass transfer in an inclined rectangular cavity filled with porous medium. International Communications in Heat and Mass Transfer, 2012. 39(2): p. 174-181.

[27] Al-Farhany, K. and A. Turan, Non-Darcy effects on conjugate double-diffusive natural convection in a variable porous layer sandwiched by finite thickness walls. International Journal of Heat and Mass Transfer, 2011. 54(13-14): p. 2868-2879.

[28] Al-Farhany, K. and A. Turan, Unsteady conjugate natural convective heat transfer in a saturated porous square domain generalized model. Numerical Heat Transfer, Part A: Applications, 2011. 60(9): p. 746-765. 